differential equation. The paper of Dr. Gillespie shows that if two known integrals, each containing $n$ dependent variables, give through the vanishing of their first variation the same system of differential equations, it is then possible to write immediately an integral of this system of differential equations.

25. The general circulation of the atmosphere has been discussed by Ferrel and Oberbeck. The former made no attempt at a complete solution. The latter assumed that the air was incompressible but satisfied Boyle's law. $\mathrm{He}$ also surrounded the atmosphere with a spherical boundary of indefinite height. In Mr. Sharpe's solution it is shown that when there is no inequality of temperature between the equator and the poles the air is in both adiabatic and conductive equilibrium. Consequently when there is an increase of temperature from the pole to the equator the temperature still satisfies Fourier's law of conduction and a circulation exists which vanishes at the upper limit of the atmosphere as well as on the earth's surface. This meridional circulation modifies the east and west motion of the air, which in turn ehanges the pressure distribution and the circulation.

$$
\begin{aligned}
& \text { F. N. Cole, } \\
& \text { Secretary. }
\end{aligned}
$$

\title{
THE DECEMBER MEETING OF THE CHICAGO SECTION.
}

THE twentieth regular meeting of the Chicago Section of the American Mathematical Society was held at the University of Chicago, Chicago, Ill., on Friday, December 28, 1906. The total attendance was thirty-five, including the following twenty-nine members of the Society :

Mr. G. D. Birkhoff, Professor G. A. Bliss, Professor Oskar Bolza, Professor D. R. Curtiss, Professor L. E. Dickson, Dr. Otto Dunkel, Professor T. F. Holgate, Mr. Louis Ingold, Professor O. D. Kellogg, Professor Kurt Laves, Mr. N. J. Lennes, Dr. A. C. Lunn, Mr. W. S. MacMillan, Professor H. Maschke, Professor G. A. Miller, Professor E. H. Moore, Dr. J. C. Morehead, Professor F. R. Moulton, Dr. L. I. Neikirk, Professor H. L. Rietz, Professor N. C. Riggs, Mr. W. J. Risley, Mr. A. R. Schweitzer, Professor J. B. Shaw, Dr. C. H. 
Sisam, Professor H. E. Slaught, Mr. R. E. Wilson, Professor J. W. A. Young, Professor Alexander Ziwet.

Two sessions were held for the reading of papers, at both of which the chairman of the Section, Professor Alexander Ziwet, presided. In the evening seventeen members of the Section dined together and enjoyer a season of social intercourse.

The committee having in charge the arrangement of a meeting for the discussion of mathematics for engineers reported that the proposed plans had been modified on account of the conflict in time with the meeting of the American association for the advancement of science in New York. In view of the announcement that this association is to meet in Chicago in December, 1907, the committee was instructed to arrange if possible a joint session of the Chicago Section with Sections $A$ and $\mathrm{D}$ of the American association and with the Society for the promotion of engineering education, which meets at the same time and place.

Professor E. B. Van Vleck was elected Chairman and Professor H. E. Slaught Secretary of the Section, for the ensuing year. These, together with Professor H. L. Rietz, constitute the programme committee; and, with the further addition of Professors A. G. Hall, E. J. Townsend, E. B. Skinner and Alexander Ziwet, constitute the committee on programme for the December, 1907, meeting.

The following papers were read:

(1) Professor James B. SHAw : "On the equivalence of algebras."

(2) Professor James B. SHAw : "Quaternion associative lineolinear expressions."

(3) Professor O. BolzA : "On Lagrange's method of multipliers in the calculus of variations."

(4) Dr. C. H. Srsam : "On a locus associated with plane curves."

(5) Professor G. A. Miller : "The groups generated by two operators such that each is transformed into its inverse by the square of the other."

(6) Mr. A. R. Schweitzer : "On an interesting class of monotonic functions."

(7) Professor L. E. Dickson : "Invariantive theory of quadratic forms in a finite field."

(8) Professor D. R. Curtiss : "Sufficient conditions for the linear dependence of functions of more than one variable." 
(9) Professor G. A. BLIss : "Note on maxima and minima of functions of several variables."

(10) Professor O. D. KelloGG: "The Green formula

$$
u(x, y)=\frac{1}{2 \pi} \int_{C} u(\sigma) \frac{\partial}{\partial \nu} G(x, y ; \xi, \eta) d \sigma . "
$$

(11) Dr. A. C. Lunn : "An example of a mechanical system with numerably infinite degrees of freedom."

Abstracts of the papers follow below, the numbers corresponding to the titles in the list above.

1. This first paper of Professor Shaw defines the equivalence of two algebras, thus: Two algebras are equivalent when, if they are supposed to have the same fundamental units, and if the product of two numbers $\alpha$ and $\beta$ is indicated for one algebra by $\Phi \cdot \alpha \beta$, for the other by $\Phi^{\prime} \cdot \alpha \beta$, then

$$
\Phi^{\prime} \cdot \alpha \beta=\phi \Phi \phi^{-1} \alpha \phi^{-1} \beta,
$$

where $\phi$ is any linear operator which transforms the units $e_{1}, \ldots, e_{r}$ into numbers $\epsilon$ given by the equations

$$
e_{i}=\phi \epsilon_{i}=\sum_{j=1}^{r} c_{i j} \epsilon_{j} \quad(i=1, \cdots, r) .
$$

It is understood that $|\phi|=\left|c_{i j}\right| \neq 0$, that is, $\phi$ has no zero roots.

The consequences of this definition are developed, with some consideration of the two problems : (1) to simplify the defining equations of an algebra by removing unessential parameters; (2) to determine algebras whose form is in some degree invariant under the transformation $\phi$, these algebras furnishing means of defining other algebras without reference to a multiplication table.

2. The second paper of Professor Shaw develops all lineolinear functions of two quaternions $q, r$ which are associative, that is, for which

$$
F(q, F[r, s])=F(F[q, r], s) .
$$

3. Professor Bolza's paper gives a proof of Lagrange's 
rule of multipliers and of the limit equations for the problem of minimizing the integral

$$
I=\int_{t_{0}}^{t_{1}} f\left(y_{1}, y_{2}, \cdots, y_{n} ; y_{1}^{\prime}, y_{2}^{\prime}, \cdots, y_{n}^{\prime}\right) d t
$$

when the admissible curves are subject to the condition of satisfying $p$ differential equations

$$
\phi_{a}\left(y_{1}, y_{2}, \ldots, y_{n} ; y_{1}^{\prime}, y_{2}^{\prime}, \ldots, y_{n}^{\prime}\right)=0 \quad(\alpha=1,2, \ldots, p)
$$

and $q$ finite equations

$$
\psi_{\beta}\left(y_{1}, y_{2}, \ldots, y_{n}\right)=0 \quad(\beta=1,2, \ldots, q),
$$

while the coördinates of their end points $\left(y_{10}, y_{20}, \ldots, y_{n 0}\right)$, $\left(y_{11}, y_{21}, \ldots, y_{n 1}\right)$ satisfy $r$ relations of the form

$$
\chi_{\gamma}\left(y_{10}, y_{20}, \ldots, y_{n 0}, y_{11}, y_{21}, \cdots, y_{n 1}\right)=0 \quad(\gamma=1,2, \ldots, r) .
$$

The proof is based upon an extension of the method used by Hilbert for the special case when $q=0$ and the end points are fixed. The paper will appear in the Mathematische Annalen.

4. Dr. Sisam's paper treats of the locus of the points of intersection of the tangents to an arbitrary algebraic curve whose points of tangency lie on a common tangent to a second algebraic curve. The order, and other interesting properties of this locus, and the corresponding properties of the dual configuration are derived. Two particular cases, first when the second of the given curves reduces to a point, and second when the locus curve coincides with the first of the two given curves, are studied in detail.

5. The main theorems proved in Professor Miller's paper may be stated as follows: If two operators neither of which is identity are such that each is transformed into its inverse by the square of the other, they must have the same order and this common order is 2,4 or 8 . If the common order exceeds two, the group generated by them involves operators of order 3 ; in fact, this group must have an $(a, 1)$ isomorphism with the symmetric group of order 24 . The smallest possible group which is generated by two such operators of order 8 is the group of order 48 known as $G_{52}$, and every other group that 
can be generated by two such operators has an $(\alpha, 1)$ isomorphism with this group of order 48 . The paper also contains a new and useful definition of the symmetric group of order 24 . This paper is to be offered to the Annals of Mathematics for publication.

6. Representing the points $0 \leqq x \leqq 1$ by the series

$$
x=\sum_{k=1}^{+\infty} \frac{\alpha_{k}}{m_{1} m_{2} \cdots m_{k}},
$$

where the numbers $\alpha_{k}, m_{k}$ are integers, $0 \leqq \alpha_{k} \leqq m_{k}-1$, $m_{k} \geqq 2$, Mr. Schweitzer discusses the function

$$
t(x)=\sum_{k=1}^{+\infty} r_{l k} \frac{\alpha_{k}^{l_{k}}}{m_{1}^{\prime} m_{2}^{\prime} \cdots m_{k}^{\prime}},
$$

where $l_{l}, m_{k}^{\prime}$ are integers. The paper will be offered to the Annals of Mathematics for publication.

7. In the paper by Professor Dickson there is established a theorem on $m$-ary quadratic forms in the $G F\left[2^{n}\right]$ analogous to that concerning the rank $r$ of the discriminantal determinant $\Delta$ in the algebraic theory and the minimum number $r$ of variables on which the form can be expressed. In place of $\Delta$ (for $m$ odd) and of all minors of odd order, we employ the halves of their algebraic expansions, necessarily with even coefficients. In particular, the semi-discriminant is an invariant. When the rank $r$ is even, there are two classes of forms, distinguishable by an arithmetic property of $\Delta^{2^{n-1}}$. Following this preliminary part, the paper proceeds to the determination and interpretation of a complete set of linearly independent invariants of the quadratic form. This invariantive theory differs in essential features from the simple algebraic theory. The paper will be offered for publication to the American Journal of Mathematics.

8. If $u_{1}, u_{2}, \cdots, u_{m}$ are $m$ functions of $n$ variables all of whose partial derivatives of the first $m-1$ orders exist and are finite throughout a given region, a necessary condition for their linear dependence is the vanishing of all $m$-rowed determinants of the matrix whose first row consists of the $m$ functions $u$, and whose succeeding rows are composed of partial derivatives of the elements of the first row, so that to each deriv- 
ative of the first $m-1$ orders there corresponds a row. In Professor Curtiss's paper some properties of matrices of this sort are developed and sufficient conditions for the linear dependence of the functions $u$ are given.

9. In investigating whether or not a function $f\left(x_{1}, x_{2}, \cdots, x_{n}\right)$ has a maximum or minimum at a point $\left(a_{1}, a_{2}, \cdots, a_{n}\right)$, it is usual to suppose that all the derivatives of the function of order less than $n$ vanish at the point $\left(a_{1}, a_{2}, \cdots, a_{n}\right)$, while one at least of order $n$ is different from zero. Then the function is expanded by Taylor's formula with a remainder to terms of order $n+1$, or else in an infinite series. In Professor Bliss's paper it is shown how the usual results in the definite and indefinite cases can be obtained from Taylor's expansion to terms of order $n$ only. The use of derivatives of higher orders than those occurring in the criteria which enable one to decide if there is a maximum or minimum is thus avoided. This paper will be offered to the BuLLETIN for publication.

10. If $u(x, y)$ be a potential function of a simply connected closed region $R$ of the $(x, y)$-plane bounded by a regular closed curve $C$, then it is known that if $u$ and its first derivatives are continuous on the boundary of $R$, it may be represented in the form

$$
u(x, y)=\frac{1}{2 \pi} \int_{C} u(\sigma) \frac{\partial}{\partial \nu} G(x, y ; \xi, \eta) d \sigma
$$

exhibiting the dependence of the function upon its boundary values $u(\sigma)$. Starting on the other hand from this formula, the question arises: under what conditions upon boundary curve $C$ and upon boundary values $u(\sigma)$ is the above integral a potential function of $R$ which approaches the boundary values $u(\sigma)$ ? For regions bounded by curves the coordinates of whose points as functions $x(s), y(s)$ of the length of arc have uniformly "algebraically continuous" derivatives (i. e., $\left|\Delta x^{\prime}(s)\right|<A|\Delta s|^{a}$, $\left|\Delta y^{\prime}(s)\right|<A|\Delta s|^{a}, 0<\alpha$ so soon as $\left.|\Delta s|<\delta\right)$, Professor Kellogg shows that a potential function is determined by a given set of boundary values through the above formula under the same conditions upon $u(\sigma)$ as in the case of the circle through Poisson's integral.

11. Dr. Lunn's paper gives the solution of an infinite system of ordinary differential equations, which are shown to determine 
the motion of an infinite linear system of discrete masses, connected by springs. The solution is obtained indirectly by a limiting process from the solution for a finite number of masses, and is then verified directly. The main features of the oscillations of a given mass are interpreted in terms of familiar properties of the Bessel functions of the time which occur as coefficients.

H. E. Slaught, Secretary of the Section.

\section{THE DECOMPOSITION OF MODULAR SYSTEMS CONNECTED WITH THE DOUBLY GEN- ERALIZED FERMAT THEOREM.}

BY PROFESSOR ELIAKIM HASTINGS MOORE.

(Read before the Chicago Section of the American Mathematical Society, December 29, 1898.)

Introduction. The Generalized Fermat Theorem $(A)$ in Purely Arithmetic Phrasing $\left(A^{\prime}, A^{\prime \prime}\right)$ with Extension $\left(A^{\prime \prime \prime}\right)$. $\$ \$ 1-5$.

1. The theorem * in question is the following:

(A) In the Galois field $G F\left[p^{n}\right]$ of prime modulus $p$ and of rank $n$ the two forms each of degree $\left(p^{n(k+1)}-1\right) /\left(p^{n}-1\right)$ in the $k+1$ indeterminates $X_{0}, X_{1}, \cdots, X_{k}$

$$
\begin{gathered}
D_{k+1, n, p}\left[X_{0}, X_{1}, \cdots, X_{k}\right]=\left|X_{j}^{p m}\right| \quad(i, j=0,1, \cdots, k) \\
P_{k+1, n, p}\left[X_{0}, X_{1}, \cdots, X_{k}\right]=\prod_{g=0, k} \prod_{a_{f g} l p^{n}}\left(X_{g}+\sum_{f=0, g-1} \alpha_{f g} X_{f}\right)
\end{gathered}
$$

are identical :

$$
D_{k+1, n, p}\left[X_{0}, \cdots, X_{k}\right]=P_{k+1, n, p}\left[X_{v}, \cdots, X_{k}\right] .
$$

Here the subscript remark $\alpha_{f g} \mid p^{n}$ indicates that the mark $\alpha_{f g}$ is to run over the $p^{n}$ marks of the Galois field $G F\left[p^{n}\right]$, and for the case $g=0$ the final $\sum_{f=0, g-1}$ does not enter.

For this theorem, which for $(k, n)=(1,1)$ is one form of Fermat's theorem, I have given three proofs, couched as is the statement of the theorem in the abstract Galois field phrasing introduced by me in the paper "A doubly-infinite system of simple groups" presented to the Chicago Congress of 1893.

* Moore, "A two-fold generalization of Fermat's theorem," Bulletin, vol. 2 (1896), pp. 189-199. 